\title{
Adaptation to altered visual-vestibular feedback: Mechanisms of maintenance and recovery
}

\author{
ROBERT B. POST \\ University of Califormia, Davis, California
}

\begin{abstract}
Adaptation of perceived movement during head motion (apparent concomitant motion, ACM) and the subsequent elimination of adaptation were studied in two experiments. During the adaptation phase of both experiments, subjects performed voluntary $1-\mathrm{Hz}$ head oscillations for 6 min while fixating a stimulus moving either in the same (with) direction as or the opposite (against) direction of head movements. In Experiment 1, ACM adaptation was measured following either a 1- or a 4-min delay after the adaptation phase. Results indicated some loss of adaptation during the additional 3-min delay, demonstrating a tendency of the system linking head and image to return to its preadaptation state following removal of an adaptation stimulus. In Experiment 2, subjects viewed a stimulus after adaptation that appeared to move minimally in the same manner as the adaptation stimulus during $3 \mathrm{~min}$ of head oscillations. No loss of adaptation was measured in these subjects between the beginning and the end of the 3-min interval. In another condition, subjects viewed a stimulus that appeared to move alternately in the same direction as and in the opposite direction of the adaptation stimulus during a similar 3-min interval following adaptation. ACM adaptation was substantially reduced during this 3-min interval. These results implicate two mechanisms that operate to either maintain or eliminate ACM adaptation. One is passive and operates in the absence of visual feedback to eliminate the short-term adapted state, and the other responds to postadaptation visual feedback.
\end{abstract}

The tendency of the visual world to remain perceptually stable during head motion is termed position constancy (Hay \& Sawyer, 1969) or constancy of visual direction (Wallach \& Kravitz, 1965a, 1965b). If the relationship between head and image motion is altered, there is a loss of position constancy, and the environment appears to move during head motion (apparent concomitant motion, $\mathrm{ACM})$. It has been known since Stratton $(1896,1897)$ that subjects adapt to ACM during prolonged exposure so that the seen motion of the environment is either diminished or eliminated. Following such adaptation, if the normal relationship between head and image motion is restored, subjects initially experience an aftereffect as the visual scene appears to move in the direction opposite that seen during adaptation. With continued observation, however, the seen motion of the visual environment disappears.

It has been proposed that the alteration of ACM from exposure to altered visual-vestibular feedback is paralleled by, and perhaps results from, modification of the vestibulo-ocular reflex (VOR) (Callan \& Ebenholtz, 1982; Hay, 1971; Post \& Lott, 1992). Specifically, when the altered relationship between head and image motion is first introduced, the gain of the VOR is inappropriate to maintain fixation on stationary objects during head rotation.

The author is grateful to Tram Dinh, Holly Graham, and Liz Warren for assistance in data collection. The author also thanks Lori Lott and Harold Bedell for providing critical feedback on an early version of the manuscript, and two anonymous reviewers for subsequent improvements. Correspondence should be addressed to R. B. Post, Department of Psychology, University of California, Davis, CA 95616 (e-mail: rbpost@ucdavis.edu).
The images of objects therefore tend to slip on the retina during head motion, and the slip may be perceived as object motion. Alternately, if subjects use the pursuit eyemovement system to maintain fixation when VOR gain is inappropriate, the pursuit activity is registered perceptually as object motion (Post \& Leibowitz, 1982). With continued exposure to the altered feedback conditions, VOR gain adapts to be more appropriate (e.g., Collewijn, Martins, \& Steinman, 1981a, 1981b; Gauthier \& Robinson, 1975; Gonshor \& Melvill-Jones, 1971). It is possible that the two proposed sources of ACM under altered feedback conditions may also provide the error signal that allows for VOR adaptation. Specifically, both retinal slip (Ito, 1982) and pursuit activity (Lisberger, Miles, \& Zee, 1984; Miles \& Lisberger, 1981a, 1981b) have been proposed to provide the basis for VOR adaptation.

The concept that the previous experience of observers influences both ACM adaptation and subsequent loss of adaptation is supported by a series of studies by Wallach and colleagues (for a discussion, see Welch 1978). For example, Wallach and Floor (1970) reported that if subjects performed a large number of head motions while viewing a stationary display prior to the introduction of altered feedback, the amount of ACM adaptation was reduced. Additionally, a few head oscillations while viewing a stationary target following adaptation led to decreases in measured adaptation (Wallach \& Frey, 1969). These findings led Wallach and colleagues to propose that observers are adapting continuously to visual field displacement during head movements in everyday life, and what is measured in the laboratory is a modification of the state achieved in daily life. It was also reported that 
when subjects are tested immediately following adaptation, and then, after a few minutes, are tested again measured adaptation is less on subsequent tests (Wallach \& Floor, 1970; Wallach \& Frey, 1969). This finding was interpreted as a tendency for the system linking head and image motion to return to a state consistent with the greatest prior experience.

Post and Graham (1994) suggested that these findings indicate the operation of two mechanisms that operate to return the head-image motion system to its preadapted state. One is passive and operates in the absence of visual input if observers merely sit in the dark. The other is driven by visual feedback from the stationary environment during postadaptation head movements. During normal head movement in a visual surround following adaptation to altered visual-vestibular feedback, both mechanisms would operate to eliminate the short-term adapted state.

The present paper was intended to explore further the mechanisms involved in either maintaining or eliminating $\mathrm{ACM}$ adaptation. The purpose of Experiment 1 was to examine whether or not spontaneous decay of the adapted state occurs during the waiting intervals. In previous studies in which adaptation decayed during waiting intervals, the interval was always placed between two adaptation measures (Wallach \& Floor, 1970; Wallach \& Frey, 1969). Therefore, the decay that occurred during the waiting interval may have been triggered by visual motion observed on the immediately preceding posttest. In the present design, the effects of delay were measured in the absence of preceding posttests.

Another purpose of Experiment 1 was to examine whether exposure to a physically stationary stimulus decreases adaptation independently of delay. In previous studies in which evidence was obtained that viewing a stationary stimulus decreased ACM adaptation, the viewing of stationary stimuli occurred between adaptation measures (Wallach \& Frey, 1969). Therefore, the loss of adaptation while viewing stationary stimuli may have resulted from passive decay, which would have otherwise occurred. In the present design, the effects of viewing stationary stimuli on ACM adaptation were measured in the absence of possible confounding effects from the passage of time involved in viewing stationary stimuli.

\section{EXPERIMENT 1}

\section{Method}

Subjects. Eight adult volunteers, $20-40$ years old, with normal oculomotor function served as subjects. The subjects were either emmetropic or mildly myopic and could focus to the distance of the stimulus display.

Apparatus. The adaptation and test stimulus was a spot of lowpower laser light that was projected onto the concave surface of a hemicylindrical screen (axis vertical) with a radius of $98 \mathrm{~cm}$. The subjects sat at the center of screen curvature (viewing distance $92 \mathrm{~cm}$ ) with their heads secured in an adjustable set of straps that was connected to a potentiometer. A voltage fed through the potentiometer and an amplifier rotated a mirror galvanometer that reflected the laser spot onto the screen, producing motion of the spot that was coupled to subjects' head motions. A potentiometer allowed either the experimenter or the subject to adjust both the amplitude (gain) and the phase of the spot motion through a range from -.6 to .6 (spot motion/head motion).

Procedure. Each subject participated in eight experimental sessions, which were separated by at least 1 day. The eight sessions represented the combination of two directions of the adaptation stimulus (with or against head motion), with two delay intervals prior to testing for adaptation ( 1 or $4 \mathrm{~min}$ ) and two initial conditions of the measurement stimulus (stationary or moving in the same direction as the adaptation stimulus). The order of sessions was counterbalanced to control for practice effects.

During each session, measures were obtained first of baseline (preadaptation) ACM. During these measures, room lights were extinguished, and the spot stimulus was adjusted to be moving slightly either with or against the subjects' head movements. The subjects then made voluntary $1-\mathrm{Hz}$ oseillatory head movements paced by an electronic metronome while fixating the stimulus and adjusted the potentiometer (and movement of the spot) so that the spot appeared stationary during the head oscillations. Post and Lott (1992) previously demonstrated that subjects could match similar metronome frequencies with head oscillations that were stable in amplitude before and after ACM adaptation. When the subjects perceived the spot as stationary, they notified the experimenter, and a 5-sec sample of both spot and head motion was collected with a microcomputer and A/D circuitry. Sampling rate was $50 \mathrm{~Hz}$. If the subjects could not achieve a setting so that the spot appeared completely stationary, they were instructed to select a setting where the perceived motion was equally with and against head motion. Three such trials were conducted, and the records of spot and head motion were stored on disk for subsequent conversion to ACM values. Examples of the sampled head and spot motions during both baseline and postadaptation trials are presented in Figure 1.

Following the measurement of baseline $\mathrm{ACM}$, the adaptation interval of the session began. The subjects performed voluntary $1-\mathrm{Hz}$ oscillations similar to those during the premeasures for $6 \mathrm{~min}$ while fixating the laser spot moving in either the same (with) direction as or the opposite (against) direction of head motion. Numerous previous studies have demonstrated significant ACM adaptation during such brief exposures (e.g., Hay \& Goldsmith, 1973; Post \& Lott, 1992, 1993; Wallach \& Kravitz, 1965b). During these oscillations, the stimulus moved through $60 \%$ of the amplitude of head motion (referenced to the stationary environment).

Following the adaptation interval, the subjects shut their eyes and sat in darkness for either 1 or $4 \mathrm{~min}$. After this delay, three consecutive measurements of ACM were obtained in the fashion described previously. The initial measurement condition was varied so that, in half of the sessions, the measurement stimulus was moving initially for each of the three measures with the same gain as the previously viewed adaptation stimulus, and subjects adjusted the gain until it was perceived as stationary; in the other sessions, the measurement stimulus was initially stationary (but perceived as moving), and the subjects adjusted the gain until it was perceived as stationary. Fivesecond samples of head and stimulus motion were then obtained and stored for each trial. All three measures were obtained in the span of $1 \mathrm{~min}$.

\section{Results}

Measures of ACM were calculated for each trial by first dividing the stored records of spot velocity by the corresponding records of head velocity. This ratio indicated the proportion of the head movement through which the spot had to move to appear stationary- that is, to nullify any ACM that was present. This nulling value was then reversed in sign to yield the magnitude of the ACM that was being canceled.

The ACM scores were subsequently used to determine a value of ACM adaptation for each of the postadapta- 


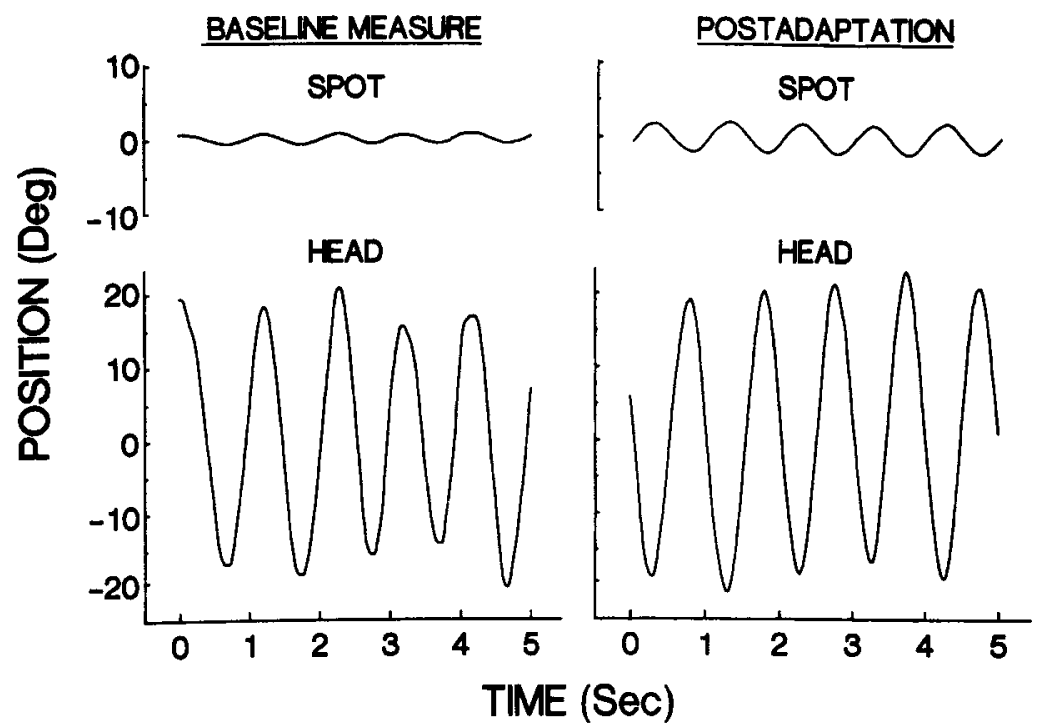

Figure 1. Representative examples of the head and spot motions measured during 5-sec ACM measurements. During a baseline measure prior to adaptation (left side), spot motion was slightly in the same direction as head motion on this particular trial. Following adaptation to an against stimulus (right side), the spot motion that appeared stationary was now opposite head motion. Interval between adaptation and test was 1 min, and the test stimulus was initially moving in the same manner as the adaptation stimulus.

tion measures. A preadaptation ACM score was first obtained by calculating the mean of the three premeasures taken in each session. This value was then subtracted from each of the postadaptation scores. The resulting difference scores represented the strength of ACM adaptation when the associated postadaptation measure was recorded. The mean of all subjects' adaptation scores are presented for each condition in Figure 2. It is apparent in the figure that adaptation scores are negative (corresponding to against motion) following with adaptation, and vice versa. Additionally, less adaptation was measured when the measurement stimulus was initially stationary than when the measurement stimulus was moving in the same direction as the adaptation stimulus. It also appears that less adaptation is measured $4 \mathrm{~min}$ after adaptation than following a 1-min delay, particularly in the case of against adaptation.

A $2 \times 2 \times 2 \times 3$ analysis of variance (ANOVA) (adaptation direction $\times$ initial measurement condition [moving or stationary] $\times$ delay interval $\times$ measure) was performed on the adaptation scores presented in Figure 2. The effect of adaptation stimulus direction apparent in Figure 2 was highly significant $[F(1,7)=73.64, p<.001]$. The main effect of delay was also statistically significant $[F(1,7)=$ $5.86, p<.05]$, with adaptation scores tending to be more negative following a 4-min delay. The interaction of adaptation direction and delay was highly significant $[F(1,7)=$ $38.45, p<.001]$, as the data for the two adaptation directions were separated further following a 1 -min delay than following a 4-min delay. Planned comparisons using the modified Bonferroni correction indicated that the adaptation was similar following a 1-and a 4-min delay in the case of with adaptation $[F(1,7)=0.70, p>$ $.1]$, whereas there was significantly less adaptation following a 4-min delay than following a 1-min delay in the case of against adaptation $[F(1,7)=37.60, p<.001]$. The interaction of adaptation direction and measure was also significant $[F(2,14)=4.04, p<.05]$, as the amount of adaptation decreased between the first and last measure. None of the other main effects or interactions was statistically significant. The interaction of initial measurement condition and adaptation direction was marginally significant $[F(1,7)=4.73, p<.1]$, as larger adaptation effects were obtained with a test stimulus that was initially moving in the same direction as the adaptation stimulus than with a test stimulus that was initially stationary.

The finding of a significant adaptation direction $\times$ delay interaction was examined further with a subsequent analysis restricted to (1) data from sessions in which the test stimulus was initially moving in the same direction as the adaptation stimulus and (2) the first of the three postadaptation test measures. The interaction again was statistically significant $[F(1,7)=7.608, p<.05]$. Therefore, less ACM adaptation is measured with a 4-min delay than with a 1-min delay following adaptation.

The nonsignificant tendency toward larger adaptation effects with a test stimulus that was initially moving in the same direction as the adaptation stimulus than with a test stimulus that was initially stationary was examined with an analysis restricted to the first of the three postadaptation test measures. The interaction was not statistically significant $[F(1,7)=1.544, p>.1]$, demonstrating that one exposure to the stationary test stimulus did not re- 


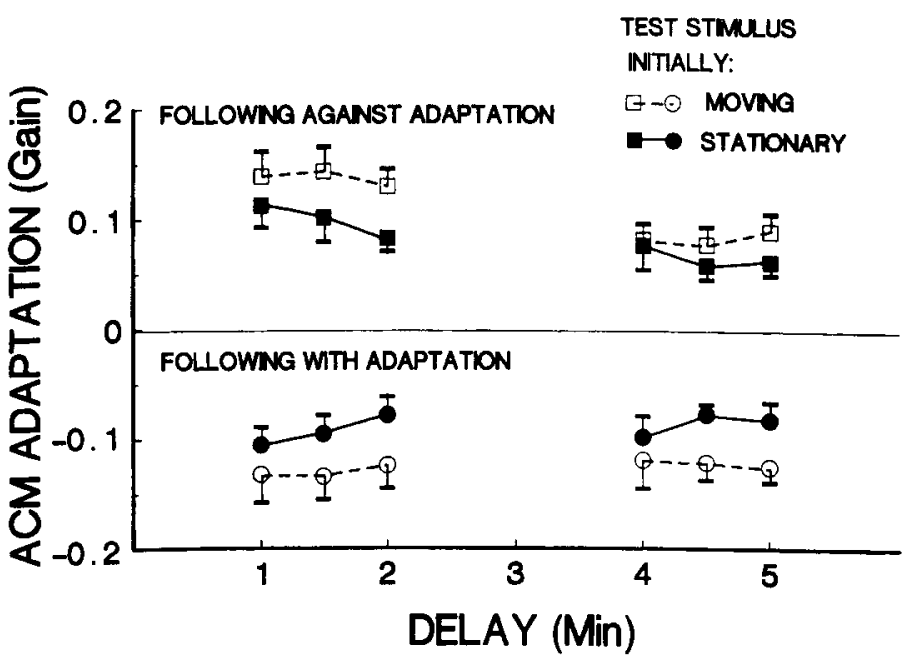

Figure 2. Change in ACM present at each postmeasure for both adaptation directions and both initial conditions of the measurement stimulus. Circles and squares present results obtained following with and against adaptation, respectively. Open symbols present results obtained when the measurement stimulus was initially moving the same way as the adaptation stimulus at the beginning of each postmeasure. Closed symbols present results obtained when the measurement stimulus was initially stationary at the beginning of each postmeasure. Error bars represent $\pm 1 S E M$. To avoid clutter, not all bars are plotted.

sult in less measured adaptation than measurements made with a stimulus that was not initially stationary. A similar analysis of the interaction of initial condition of the test stimulus $\times$ adaptation direction was then conducted on the data from the third of the three postadaptation test measures. The interaction restricted to these measures was statistically significant $[F(1,7)=9.881, p<.05]$. Therefore, after three exposures to a test stimulus that is initially stationary, less ACM adaptation is measured than when the test stimulus is initially moving in the direction of adaptation.

\section{Discussion}

The 6-min exposure to a stimulus moving concomitantly with head oscillations produced ACM adaptation. Stimuli moving with the head produced against ACM, whereas stimuli moving opposite the head produced with $\mathrm{ACM}$. These results are consistent with a number of previous studies of these effects (e.g., Hay, 1968; Post \& Lott, 1992, 1993; Stratton, 1896; Tietz \& Gogel, 1978; Wallach \& Kravitz, 1965a, 1965b).

ACM adaptation was greater following a 1-min delay than following a 4-min delay. This finding suggests that the system linking head and image motion has a tendency to return to its preadaptation state after removal of an adaptation stimulus in the absence of subsequent visual input. Previous studies (Wallach \& Frey, 1969; Wallach \& Floor, 1970 ) reported that a delay interposed between two tests for $\mathrm{ACM}$ adaptation resulted in less measured adaptation on the second of the two tests. Although these findings were interpreted as a tendency to return to the adaptation state consistent with the greatest prior experience, the effects of delay were never assessed without a preceding test for adaptation. It is therefore possible that the measured loss of adaptation was completely triggered or to some degree triggered by the preceding test. In the present experiment, evidence for a passive "decay" mechanism was obtained without the potential interfering effects of a preceding test.

The results of Experiment 1 may indicate that VOR gain adaptation is prone to spontaneous decay as well, if it is assumed that the ACM changes parallel VOR gain alterations (e.g., Callan \& Ebenholtz, 1982; Hay, 1971; Post \& Lott, 1992). Evidence consistent with this possibility was reported by Miles and Eighmy (1980) in a study of long-term VOR modification in the monkey. Although the changes in the present experiment were tracked over a shorter time interval, it is interesting to note that both the present ACM adaptation and the VOR modification measured by Miles and Eighmy (1980) decayed only following against adaptation conditions, which produce VOR gain enhancement.

Measured ACM adaptation tended to be larger when the test stimulus was initially moving in the same direction as the adaptation stimulus than when it was initially physically stationary. It is expected that exposure to a stationary stimulus results in less measured ACM adaptation than do trials where subjects view a test stimulus that initially resembles the adaptation stimulus, if one considers viewing the stationary stimulus as commencing a new adaptation. That is, following adaptation of ACM (and the VOR), viewing a stationary stimulus exposes the subject to retinal slip in the direction opposite that of the adaptation stimulus, and this slip (or the elicited pursuit effort) provides the basis for re-adaptation.

Wallach and Frey (1969) had previously reported that head oscillations while viewing a stationary stimulus de- 
creased the amount of adaptation measured between one posttest and the next. Although these findings were interpreted as a tendency for the system to readapt to a prior familiar state when exposed to it, the effects of viewing a stationary stimulus were not compared with a condition wherein subjects waited passively between measures. In the present experiment, evidence for the role of a mechanism that is driven by visual feedback was obtained without the potential confounding effects of an intervening delay. It cannot be determined whether the fact that the stationary stimulus viewed resembles extensive prior experience of the subjects (i.e., a visual stimulus that is physically stationary during head motion) is of special importance in returning the head-image motion system to its preadaptation condition. It is possible that subjects would rapidly adapt to other head-image motion relationships after an initial adaptation, independent of whether they were familiar or not. Therefore, there is no basis for evaluating the conclusion of Wallach and colleagues that prior familiarity is an important feature of the readaptation.

In sum, Experiment 1 provided evidence for the operation of two mechanisms for the resetting of the headimage motion system to its preadaptation condition following adaptation to altered visual-vestibular feedback. One is passive and operates if subjects merely sit in the dark following adaptation. The other is dependent on visual feedback during head motion following adaptation.

\section{EXPERIMENT 2}

In Experiment 1, viewing a stationary stimulus during head oscillations following ACM adaptation decreased the amount of measured adaptation. Although this stimulus was physically stationary, it was seen to be moving in the direction opposite the preceding adaptation stimulus. This finding raises the possibility that seen motion, from either retinal slip or pursuit activity, is essential for either ACM adaptation or the resetting of the system following adaptation. In Experiment 2, the characteristics of seen motion following an adaptation interval were manipulated to determine its effect on adaptation in two different conditions.

\section{Method}

Subjects and Apparatus. The subjects and the apparatus were the same as those in Experiment 1.

Procedure. Each subject participated in four sessions, representing the combination of two directions of adaptation motion with two experimental conditions: minimal motion and oscillatory motion.

Minimal motion. The subjects adapted for $6 \mathrm{~min}$ to a stimulus moving either with or against the head in the same manner as in Experiment 1 . At the end of this adaptation period, the subjects adjusted the potentiometer so that the spot appeared stationary, and a sample of ACM was obtained. The subjects then adjusted the spot so that it appeared to be moving just perceptibly in the same direction as the adaptation stimulus and then viewed this stimulus for $3 \mathrm{~min}$ while continuing to make head oscillations. At the end of this 3-min interval, the subjects again adjusted the potentiometer so that the spot appeared stationary, and another sample of ACM was taken.

Oscillatory motion. The oscillatory motion condition was identical to the minimal motion condition with the exception of the stim- ulus viewed during the 3-min interval separating the two postadaptation ACM measures. Instead of viewing a stimulus that appeared to be moving in the same direction as the adaptation stimulus, the subjects continuously adjusted the stimulus so as to appear to move alternately in the same direction as and the opposite direction of adaptation motion. These adjustments were made throughout the 3 min postadaptation interval. In this way, the subjects were exposed to equal amounts of motion perceived in the same direction as or the opposite direction of head motion, if it is assumed that it took the same time to adjust from same to opposite motion as it did to make the reverse adjustment.

\section{Results}

Because the motion stimuli viewed during the 3-min postadaptation interval were determined by the subjects' potentiometer adjustments, initial analyses were conducted to characterize the stimuli that the subjects had selected. In the minimal motion condition, after the subjects had made the initial postadaptation setting, they increased the gain of spot motion by a mean value of $.12(S D=.05)$ to produce reliable with seen motion. For against adaptation sessions, the corresponding mean change of spot motion was .14 in the negative direction $(S D=.07)$ to produce reliable perceived against motion. These values are approximately $20 \%-23 \%$ of the \pm .60 gain of the adaptation stimulus.

In the oscillatory motion condition, the mean number of cycles of alternating with and against motions viewed during the interval following adaptation was 15.6 in with adaptation sessions and 16.2 in against adaptation sessions. The mean gains of the stimuli viewed were .095 $(S D=.086)$ and $-.147(S D=.057)$ following with and against adaptation, respectively. The subjects viewed with motion following with adaptation $68.2 \%$ of the $3 \mathrm{~min}$, and they viewed against motion following against adaptation $85.6 \%$ of the $3 \mathrm{~min}$. These analyses indicate that the stimulus viewed during the 3 -min postadaptation interval was moving predominantly in the same direction as the adaptation stimulus, although it was perceived to be moving in the direction opposite the adaptation stimulus half the time.

The gain settings selected by the subjects to correspond to no $\mathrm{ACM}$ before and after the 3-min postadaptation interval are presented for both the minimal motion and the oscillatory motion conditions in Figure 3. It is apparent in the figure that the no motion settings correspond to with and against motion following with and against adaptation, respectively, for both conditions. For the minimal motion condition, the scores from the end of the $3 \mathrm{~min}$ tend to be somewhat more negative than do those from the beginning of the interval. There does not, however, appear to be an overall loss of adaptation between the beginning and end of the $3 \mathrm{~min}$. In the oscillatory motion condition, there appears to be a loss of adaptation between the beginning and end of the 3-min postadaptation interval, since the scores from both with and against adaptation sessions are closer to zero at the end of the 3 min than at the beginning.

Separate $2 \times 2$ ANOVAs (direction of adaptation $\times$ beginning vs. end of postadaptation interval) were per- 


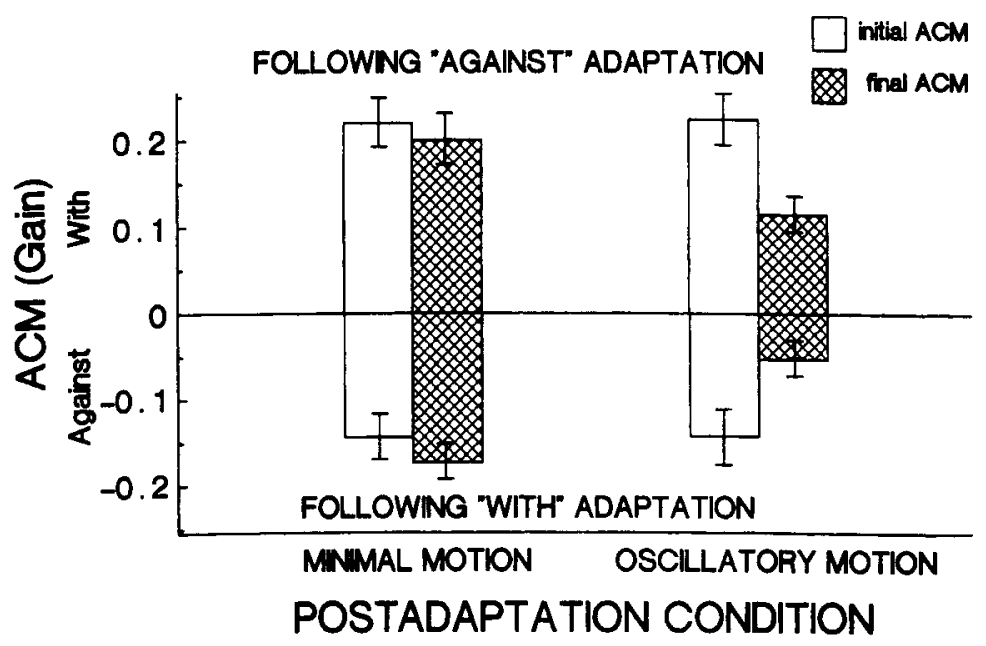

Figure 3. ACM adaptation before and after 3-min postadaptation intervals for the minimal motion and oscillatory motion conditions of Experiment 2. Error bars represent $\pm 1 S E M$.

formed for the minimal motion and oscillatory motion conditions on the values presented in Figure 3. For the minimal motion condition, the effect of adaptation stimulus direction apparent in Figure 3 was highly significant $[F(1,7)=70.81, p<.001]$. The main effect of beginning versus end of postadaptation interval was also statistically significant $[F(1,7)=5.60, p<.05]$, the with adaptation scores tending to be more negative following a 3min delay. The interaction of direction of adaptation and beginning versus end of interval was not statistically significant $[F(1,7)=0.18, p>.1]$. For the oscillatory motion condition, the main effect of adaptation direction was highly significant $[F(1,7)=55.00, p<.001]$. The interaction of adaptation direction and beginning versus end of interval was also statistically significant $[F(1,7)=$ $24.40, p<.01]$, as null ACM values were shifted further in the direction of the adaptation stimulus at the beginning of the 3-min postadaptation interval than at the end. Planned comparisons using the modified Bonferroni correction were made separately for the results obtained for with and against adaptation in the oscillatory motion condition. The decay of ACM toward zero between the beginning and the end of the 3-min postadaptation interval was significant for both the with adaptation sessions $[F(1,7)=5.94, p<.05]$ and the against adaptation sessions $[F(1,7)=14.50, p<.01]$.

\section{Discussion}

The results of the minimal motion condition of Experiment 2 demonstrated that exposure to a stimulus perceived to be moving in the same direction as the adaptation stimulus during the 3-min interval following adaptation prevented the decay of adaptation that otherwise occurs. The motion observed was selected by the subjects to be just perceptibly in the same direction as the adaptation stimulus and therefore represented a small percentage of the magnitude of the adaptation stimulus. Nevertheless, the seen motion was sufficient to prevent or override the spontaneous decay of adaptation that occurs in the absence of stimulation.

The results of the oscillatory motion condition demonstrated that exposure to a stimulus perceived to be moving alternately in the same direction as and the opposite direction of the preceding adaptation stimulus decreased ACM adaptation substantially. It is possible that when perceived motion is equivalent following adaptation, the situation is similar to that observed in darkness, and the passive mechanism manifests itself. Alternatively, it is possible that, although the subject is exposed to equal amounts of motion perceived in the same direction as and the opposite direction of the adaptation stimulus, the system is predisposed to adjust itself to the pattern of motion with which it has the greatest prior experience. Therefore, the motion perceived as opposite the prior adapting stimulus corresponds more closely to the relationship between head and image motion with which the subject has vastly more prior experience. It has been suggested previously (e.g., Wallach \& Floor, 1970; Wallach \& Frey, 1969) that the head-image movement system has a bias to return to a state with which it has a large amount of prior experience. The present results support this hypothesis.

It should be remembered that the perceived motion of the stimuli viewed during the postadaptation interval in Experiment 2 was the result of either retinal slip or pursuit activity. It is possible that the perception of motion, per se, is irrelevant to the effects of the minimal motion and oscillatory motion conditions on ACM. Rather, the retinal slip characteristics or pursuit requirements of the stimuli may have been the critical element for maintaining or eliminating ACM adaptation.

It is important to note that, in the oscillatory motion condition, although subjects perceived equal amounts of motion in the same direction as and the opposite direction of the adaptation stimulus, they actually were exposed for the most part to a stimulus moving in the same manner as the adaptation stimulus. The finding that sub- 
stantial decreases in ACM adaptation occurred despite continued exposure to a stimulus resembling the adaptation stimulus to some extent suggests that the perceived direction of motion is of relatively greater importance in maintaining or eliminating an adapted state than are the physical characteristics of stimulus motion.

The findings of the oscillatory motion condition are consistent with the idea that viewing a pattern of motion similar to that experienced previously is especially influential in determining the state of the head-image motion system. This finding may relate to the tolerance displayed by experienced spectacle wearers for two relationships between head and image motion. With repeated exposures to an altered relationship between head and image motion, the altered state acquires "familiar" status. Once dual familiar states are established, some form of discriminative cue is required for the system to switch between states. Shelhamer, Robinson, and Tan (1992) have shown that subjects can develop different VOR gains that are contingent on gaze angle. The present results may suggest that the small amounts of seen motion, retinal slip, or pursuit activity during initial head movements following putting on or removing glasses provide cues for the system to rapidly switch states, independent of other cues, such as the presence of frames.

\section{REFERENCES}

Callan, J. W., \& Ebenholtz, S. M. (1982). Directional changes in the vestibular ocular response as a result of adaptation to optical tilt. $V i$ sion Research, 22, 37-42.

Collewijn, H., Martins, A. J., \& Steinman, R. M. (1981a). Natural retinal image motion: Origin and change. In B. Cohen (Ed.), Vestibular and oculomotor physiology: International meeting of the Barany Society (Annals of the New York Academy of Sciences, Vol. 374, pp. 312-329). New York: New York Academy of Sciences.

Collewijn, H., Martins, A. J., \& Steinman, R. M. (1981b). The time course of adaptation of human compensatory eye movements. In L. Maffei (Ed.), Pathophysiology of the human visual system (Documenta Ophthalmologica Proceedings Series, Vol. 30, pp. 123-133). The Hague: W. Junk.

GaUthier, G. M., \& RoBinson, D. A. (1975). Adaptation of the human vestibuloocular reflex to magnifying lenses. Brain Research, 92, 331335.

Gonshor, A., \& Melvill-Jones, G. (1971). Plasticity in the adult human vestibulo-ocular reflex arc. Proceedings of the Canadian Federation of Biological Science, 14, 11.

HAY, J. C. (1968, April 26). Visual adaptation to an altered correlation between eye movement and head movement. Science, 160, 429-430.

HAY, J. C. (1971). Does head-movement feedback calibrate the perceived direction of optical motions? Perception \& Psychophysics, 10, 286-288.

HAY, J. C., \& Goldsmith, W. M. (1973). Space-time adaptation of visual position constancy. Journal of Experimental Psychology, 99, 1-9.
HAY, J. C., \& SAWYER, S. (1969). Position constancy and binocular convergence. Perception \& Psychophysics, 5, 310-312.

ITo, M. (1982). Cerebellar control of the vestibulo-ocular reflex around the flocculus hypothesis. Annual Review of Neuroscience, 5, 275-296.

Lisberger, S. G., Miles, F. A., \& ZeE, D. S. (1984). Signals used to compute errors in monkey vestibuloocular reflex: A possible role of flocculus. Journal of Neurophysiology, 52, 1140-1153.

Miles, F. A., \& EIGHMY, B. B. (1980). Long-term adaptive changes in primate vestibuloocular reflex: I. Behavioral observations. Journal of Neurophysiology, 43, 1406-1425.

MiLES, F. A., \& LisBerger, S. G. (1981a). The "error" signals subserving adaptive gain control in the primate vestibulo-ocular reflex. In B. Cohen (Ed.), Vestibular and oculomotor physiology: International meeting of the Barany Society (Annals of the New York Academy of Sciences, Vol. 374, pp. 513-525). New York: New York Academy of Sciences.

Miles, F. A., \& Lisberger, S. G. (1981b). Plasticity in the vestibuloocular reflex: A new hypothesis. Annual Review of Neuroscience, 4, 273-299.

Post, R. B., \& Graham, H. H. (1994). Adaptation to altered visualvestibular feedback: Roles of seen movement and exposure history. Investigative Ophthalmology \& Visual Science, 35, 2036.

Post, R. B., \& Leibowitz, H. W. (1982). The effect of convergence on the vestibulo-ocular reflex and implications for perceived movement. Vision Research, 22, 461-465.

PosT, R. B. \& LoTT, L. A. (1992). The relationship between vestibuloocular reflex plasticity and changes in apparent concomitant motion. Vision Research, 32, 89-96.

Post, R. B., \& LotT, L. A. (1993). Frequency specificity in the adaptation of apparent concomitant motion. Bulletin of the Psychonomic Society, 31, 53-56.

Shelhamer, M., Robinson, D. A., \& TAN, H. S. (1992). Context-specific adaptation of the gain of the vestibulo-ocular reflex in humans. Journal of Vestibular Research, 2, 89-96.

STRATTON, G. (1896). Some preliminary experiments on vision without inversion of the retinal image. Psychological Review, 3, 611-617.

Stratton, G. (1897). Vision without inversion of the retinal image. Psychological Review, 4, 341-360, 463-481.

TIETZ, J. D., \& Gogel, W. C. (1978). Adaptation to apparent concomitant motion in the absence of physical or retinal motion. Bulletin of the Psychonomic Society, 12, 1-4.

WALLACH, H., \& FLOOR, L. (1970). On the relation of adaptation to field displacement during head movements to the constancy of visual direction. Perception \& Psychophysics, 8, 95-98.

Wallach, H., \& FREY, K. J. (1969). Adaptation in the constancy of visual direction measured by a one-trial method. Perception \& Psychophysics, 5, 249-252.

Wallach, H., \& Kravitz, J. H. (1965a). The measurement of the constancy of visual direction and of its adaptation. Psychonomic Science, 2, 217-218.

WALLACH, H., \& KRAVITZ, J. H. (1965b). Rapid adaptation in the constancy of visual direction with active and passive rotation. Psychonomic Science, 3, 165-166.

WelCH, R. B. (1978). Perceptual modification. New York: Academic Press.

(Manuscript received December 1, 1995; revision accepted for publication March 25, 1996.) 